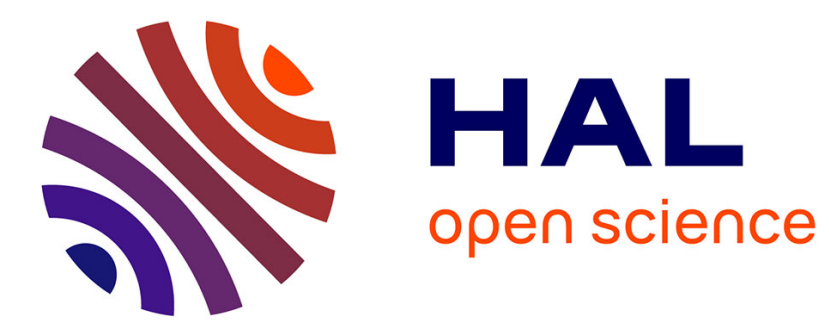

\title{
Sodium-glucose co-transporter inhibitors in insulin-treated diabetes: a meta-analysis
}

\author{
João Pedro Ferreira, Ana Cristina Oliveira, Francisca Saraiva, Francisco
}

Vasques-Nóvoa, Adelino Leite-Moreira

\section{- To cite this version:}

João Pedro Ferreira, Ana Cristina Oliveira, Francisca Saraiva, Francisco Vasques-Nóvoa, Adelino Leite-Moreira. Sodium-glucose co-transporter inhibitors in insulin-treated diabetes: a meta-analysis. European Journal of Endocrinology, 2021, 184 (6), pp.783-790. 10.1530/EJE-20-1484 . hal-03364314

\section{HAL Id: hal-03364314 \\ https://hal.univ-lorraine.fr/hal-03364314}

Submitted on 4 Oct 2021

HAL is a multi-disciplinary open access archive for the deposit and dissemination of scientific research documents, whether they are published or not. The documents may come from teaching and research institutions in France or abroad, or from public or private research centers.
L'archive ouverte pluridisciplinaire HAL, est destinée au dépôt et à la diffusion de documents scientifiques de niveau recherche, publiés ou non, émanant des établissements d'enseignement et de recherche français ou étrangers, des laboratoires publics ou privés. 
1 Sodium-glucose co-transporter inhibitors in insulin-treated diabetes: a meta-analysis

2

3 Authors:

4 João Pedro Ferreira, MD, $\mathrm{PhD}^{1,2}$; Ana Cristina Oliveira, $\mathrm{Msc}^{2}$; Francisca A. Saraiva, Msc ${ }^{2}$;

$5 \quad$ Francisco Vasques-Nóvoa, MD, $\mathrm{Msc}^{2}$; Adelino Leite-Moreira, MD, $\mathrm{PhD}^{2}$

6

7 Affiliations :

$8{ }^{1}$ Université de Lorraine, Inserm, Centre d'Investigations Cliniques, - Plurithématique 14-33,

9 and Inserm U1116, CHRU, F-CRIN INI-CRCT (Cardiovascular and Renal Clinical Trialists),

10 Nancy, France.

$11{ }^{2}$ Cardiovascular Research and Development Center, Department of Surgery and Physiology,

12 Faculty of Medicine of the University of Porto, Porto, Portugal.

13

14 Contact to:

15 Dr João Pedro Ferreira

16 Centre d'Investigation Clinique 1433 module Plurithématique

17 CHRU Nancy - Hopitaux de Brabois, Institut Lorrain du Coeur et des Vaisseaux Louis Mathieu

184 rue du Morvan, 54500 Vandoeuvre les Nancy

19 Tel : +33(0) 383157315

$20 \quad$ Fax : +33(0) 383157324

$21 \quad$ Mail: j.ferreira@chru-nancy.fr 
Abstract

Background: Patients with insulin-treated type 2 diabetes (T2D) have a high risk of major adverse cardiovascular events. Sodium-glucose cotransporter inhibitors (SGLTi) improve outcomes without hypoglycemic risk.

Aims: To study the effect of SGLTi in patients with T2D with and without background insulintreatment in outcome-driven RCTs.

Methods: Random effects models.

Results: A total of 54,374 patients with T2D were included in the analysis, of which 26,551 $(48.8 \%)$ were treated with insulin. For 3P-MACE in patients without insulin treatment, the HR $(95 \% \mathrm{Cl})$ for the effect of SGLTi vs. placebo was $0.93(0.81-1.05)$, with moderate heterogeneity $(12=49.2 \%, Q$ statistic $P=0.11)$. In insulin-treated patients, the $\mathrm{HR}(95 \% \mathrm{Cl})$ was $0.88(0.82-0.95)$, without evidence of heterogeneity $(I 2=0.0 \%, Q$ statistic $P=0.91)$. The pooled effect evidenced a $10 \%$ reduction of 3P-MACE with SGLTi (HR 0.90, 95\%CI 0.85-0.96), without SGLTi-by-insulin interaction $\mathrm{P}=0.53$. For the composite outcome of $\mathrm{HF}$ hospitalisation or cardiovascular death in patients without insulin treatment, the HR $(95 \% \mathrm{Cl})$ for the effect of SGLTi vs. placebo was 0.77 (0.61-0.92), with marked heterogeneity (I2 $=66.8 \%, Q$ statistic $P=0.02)$. In insulin-treated patients, the $H R(95 \% \mathrm{Cl})$ was $0.77(0.68-0.86)$, without significant heterogeneity $(12=31.7 \%$, $Q$ statistic $P=0.25)$. The pooled effect evidenced a $23 \%$ reduction of HF hospitalisations or cardiovascular death with SGLTi (HR $0.77,95 \% \mathrm{Cl} 0.68-0.85)$, without SGLTi -by-insulin interaction $\mathrm{P}=0.98$

Conclusion: SGLTi reduce cardiovascular events regardless of insulin use. However, the treatment effect is more homogeneous among insulin-treated patients, supporting the use of SGLTi for the treatment of patients with T2D requiring insulin for glycemic control. 
47 Key-words: type 2 diabetes; insulin; sodium-glucose cotransporter 2 inhibitors;

48 cardiovascular outcomes; meta-analysis.

49

50 
Patients with insulin-treated diabetes have a high risk of major adverse cardiovascular events (MACE), frequently leading to symptomatic heart failure (HF), hospitalizations and death $^{1}$. The risk of patients with type 2 diabetes (T2D) treated with insulin is higher than the risk of patients with T2D treated with oral anti-diabetic drugs only (i.e., non-insulin-treated diabetes $)^{2-4}$. In patients with T2D, insulin treatment is associated with long-standing diabetes, poor cardiometabolic profile (higher body mass index, abdominal obesity, heart rate, blood pressure, triglycerides and lower HDL cholesterol) and a higher prevalence of micro- and macrovascular complications ${ }^{5,6}$. Sodium-glucose cotransporter $1 / 2$ or 2 inhibitors (SGLTi) have demonstrated to improve cardiovascular and kidney outcomes in patients with T2D and in patients with heart failure with reduced ejection fraction (HFrEF) with and without diabetes ${ }^{7-11}$. The reduction of hospitalisations due to $\mathrm{HF}$ is particularly striking with these agents ${ }^{12,13}$. As patients with insulin-treated diabetes have a high-risk of cardiovascular complications, including HF, it is plausible that SGLTi provide major benefits to these patients by potentially reducing their risk of MACE, including HF hospitalisations and cardiovascular death. Furthermore, SGLTi may help controlling blood pressure, weight, and sodium retention, thus counteracting the deleterious effects associated with insulin-treatment without increasing the risk of hypoglycemic episodes ${ }^{14-20}$. Using updated data from large outcome-driven randomised controlled trials (RCTs) assessing the efficacy and safety of SGLTi versus placebo, we aim to study the effect of these agents in patients with T2D with and without background insulin-treatment.

\section{Methods}




\section{Search strategy and selection criteria}

This meta-analysis was conducted in accordance with the Preferred Reporting Items for Systematic Reviews and Meta-analyses (PRISMA) reporting guideline ${ }^{21}$. We defined both MeSH terms (controlled language) and free text terms to express each component of PICO expression: P) Population, patients with diabetes; I) Intervention, SGLT inhibitors; C) Comparison, placebo and O) Outcomes, MACEs. The search query included the following and it was last searched on $22^{\text {nd }}$ of November 2020: ("Diabetes Mellitus"[Mesh] OR diabetes) AND ("Sodium-Glucose Transporter 2 Inhibitors"[Mesh] OR sglt inhibitors) AND ("Placebos"[Mesh] OR placebo) AND ("Randomized Controlled Trial" [Publication Type] OR randomized controlled trial) AND (cardiovascular death OR cardiovascular events). We did not restrict the search by language, date or publication status. Additionally, we have included recently published trials that were not displayed on PubMed at the time ${ }^{22,23}$. One hundred fifteen studies were initially identified, of which 108 were excluded because of inadequate study design, outcomes or topic of interest. Then we have assessed whether the identified trials displayed subgroup analyses in patients with and without background insulin treatment, resulting in 6 selected RCTs: 1) Empagliflozin Cardiovascular Outcome Event Trial in Type 2 Diabetes Mellitus Patients (EMPA-REG OUTCOME) ${ }^{7} ; 2$ ) the CANVAS-Program, consisting of the Canagliflozin Cardiovascular Assessment Study (CANVAS) and A Study of the Effects of Canagliflozin on Renal Endpoints in Adult Participants With Type 2 Diabetes Mellitus (CANVAS-R) ${ }^{8}$; 3) the Multicenter Trial to Evaluate the Effect of Dapagliflozin on the Incidence of Cardiovascular Events (DECLARE-TIMI 58) ${ }^{9}$; 4) the Cardiovascular Outcomes Following Ertugliflozin Treatment in Type 2 Diabetes Mellitus Participants With Vascular Disease $\left(\right.$ VERTIS-CV) $\left.{ }^{24} ; 5\right)$ the Effect of Sotagliflozin on Cardiovascular Events in Patients With Type 2 Diabetes Post Worsening Heart Failure (SOLOIST-WHF) ${ }^{22}$; and the 6) Effect of 
Sotagliflozin on Cardiovascular and Renal Events in Patients With Type 2 Diabetes and Moderate Renal Impairment Who Are at Cardiovascular Risk (SCORED) ${ }^{23}$. Trial eligibility was confirmed by 2 independent reviewers (J.P.F., A.C.O.), data extraction was performed by A.C.O and J.P.F, and the study quality was performed by J.P.F., A.C.O., and F.A.S. We have also cross-checked the RCTs selected to the present analysis with other recently published meta-analyses and the concordance was perfect ${ }^{12,13,25}$. The Evaluation of the Effects of Canagliflozin on Renal and Cardiovascular Outcomes in Participants With Diabetic Nephropathy (CREDENCE) ${ }^{10}$, which was primarily a kidney outcomes trial, did not report treatment effects in insulin subgroups and was not included in the analysis. A detailed description of each individual trial is provided in the respective main reports as above referenced.

The analyses were performed to assess heterogeneity of treatment effects in outcomes in patients with and without background insulin treatment.

\section{Outcomes}

The primary outcome in EMPA-REG OUTCOME, CANVAS-Program, DECLARE-TIMI 58, and VERTIS-CV was the composite of time-to-first of myocardial infarction, stroke, or cardiovascular death (3P-MACE). The primary outcome in SOLOIST-WHF and SCORED was the total number of deaths from cardiovascular causes and hospitalizations and urgent visits for HF (first and recurrent). On top of the primary outcome, the EMPA-REG OUTCOME trial reported the treatment effect of empagliflozin in patients with and without insulin treatment for the outcome of cardiovascular death. The DECLARE-TIMI 58 and VERTIS-CV reported the treatment effect of dapagliflozin and ertugliflozin, respectively, in patients with and without insulin treatment for the composite outcome of time-to-first of cardiovascular death or HF hospitalisation. For optimizing the consistency of outcomes across trials, the 

infarction, stroke, or cardiovascular death. The EMPA-REG OUTCOME, DECLARE-TIMI 58, VERTIS-CV, SOLOIST-WHF, and SCORED trials were meta-analyzed for the outcomes of cardiovascular death (EMPA-REG OUTCOME) and composites of cardiovascular death or HF hospitalisations, as time-to-first event (DECLARE-TIMI 58 and VERTIS-CV) or first and recurrent (SOLOIST-WHF and SCORED). The studied trials and outcomes are displayed in Table 1.

\section{Statistical analysis}

A random-effect meta-analysis approach was used with heterogeneity assessed using the Cochran $Q$ test statistic and Higgins and Thompson $12^{26}$. Heterogeneity was considered to be low, moderate, or high if 12 was $<25 \%, 25 \%$ to $75 \%$, or $>75 \%$, respectively. The hazard ratio $(\mathrm{HR})$ and respective $95 \%$ confidence intervals $(95 \% \mathrm{Cl})$ were extracted from the insulin subgroup analyses reported in the publications of each individual study. All studies, except DECLARE-TIMI 58, reported the HR $(95 \% \mathrm{Cl})$ for patients with and without insulin treatment. In DECLARE-TIMI 58 we have extracted the approximate $\mathrm{HR}(95 \% \mathrm{Cl})$ from the forest plots.

The number and proportion of patients with events in insulin subgroups were inconsistently reported and we show these numbers in a table whenever available. The meta-analysis was directly implemented on the HR (exponential) scale. The assessment of whether background insulin treatment could modify the effect of SGLTi on outcomes was performed using interaction term for subgroups and reported with a P-value for interaction. Records identified in each database were imported and managed through Microsoft Excel ${ }^{\circledR}$. We assessed publication bias by visual inspection of funnel plots, with the ascertainment for potential asymmetry of published results by Egger's regression test and Duval and Tweedie's 
trim-and-fill method. The statistical analyses were performed using Stata ${ }^{\circledR}$ software version 16 (StataCorp. 2019. Stata Statistical Software: Release 16. College Station, TX: StataCorp LLC.) and the package meta. The risk of bias in randomised trials was assessed with the RoB 2 tool $^{27}$. Two-sided $\mathrm{P}$ values $<0.05$ were considered significant.

\section{Results}

The characteristics of the patients from the RCTs included in this analysis are depicted in the Table 2. A total of 54374 patients with T2D were included in the analysis, of which 26551 (48.8\%) were treated with insulin. The mean age of all trial participants was 66 years, 19702 (36.2\%) were women, and 43806 (80.6\%) were Caucasian. The number of participants per trial ranged from 1222 in SOLIST-WHF to 17160 in DECLARE-TIMI 58. The median follow-up ranged from 0.8 years in SOLIST-WHF to 4.2 years in DECLARE-TIMI 58 (Table 2). The assessment of risk of bias by visual inspection of funnel plots and quantitative assessment suggested no indication of publication bias. The risk of bias in randomized trials as assessed by the RoB 2 tool was low.

The number of events per trial and insulin subgroup are presented in the Table 3. The treatment effect of SGLTi vs. placebo in patients with and without insulin treatment for the composite outcome of myocardial infarction, stroke or cardiovascular death and the composite of heart failure hospitalisation or cardiovascular death (cardiovascular death alone in the EMPA-REG OUTCOME trial) are presented in the Table 3 and Figure 1.

For the composite outcome of myocardial infarction, stroke or cardiovascular death in patients without insulin treatment, the $\mathrm{HR}(95 \% \mathrm{Cl})$ for the effect of SGLTi vs. placebo was $0.93(0.81-1.05)$, with moderate heterogeneity $(I 2=49.2 \%, Q$ statistic $P=0.11)$. In insulintreated patients, the HR $(95 \% \mathrm{Cl})$ for the effect of SGLTi vs. placebo was $0.88(0.82-0.95)$, 
without evidence of heterogeneity $(12=0.0 \%, Q$ statistic $P=0.91)$. The pooled effect evidenced a $10 \%$ reduction of 3P-MACE with SGLTi (HR 0.90, 95\%Cl 0.85-0.96), without significant interaction between subgroups of insulin (yes vs. no), interaction $P=0.53$. Figure 1, panel A.

For the composite outcome of HF hospitalisation or cardiovascular death (cardiovascular death alone in the EMPA-REG OUTCOME trial) in patients without insulin treatment, SGLTi reduced the event rate by $23 \%$ (HR $0.77,95 \% \mathrm{Cl} 0.61-0.92)$ but the treatment effect was heterogeneous ( $12=66.8 \%$, $Q$ statistic $P=0.02)$. In insulin-treated patients, SGLTi significantly reduced HF hospitalisation or cardiovascular death (HR 0.77, $95 \% \mathrm{Cl} 0.68-0.86)$ without significant heterogeneity $(12=31.7 \%$, $Q$ statistic $P=0.25)$. The pooled effect evidenced a $23 \%$ reduction of HF hospitalisations or cardiovascular death with SGLTi (HR 0.77, 95\%Cl 0.68-0.85), without significant interaction between subgroups of insulin (yes vs. no), interaction $\mathrm{P}=0.98$. Figure 1 , panel $B$.

\section{Discussion}

The present meta-analysis shows that SGLTi reduced the composite outcome of myocardial infarction, stroke or cardiovascular death and the composite outcome of HF hospitalisation or cardiovascular death regardless of insulin use at baseline. However, the treatment effect was more homogeneous and consistent among insulin-treated patients, highlighting the need for adding a SGLTi to the therapeutic armamentarium of patients with T2D requiring insulin for glycemic control.

In patients with T2D, insulin is used to lower blood glucose levels in patients that fail to achieve targets or that do not tolerate oral glucose-lowering therapies. However, the intensity of glucose lowering should be tailored to each individual patient as it may increase 
hypoglycemic episodes and mortality ${ }^{28,29}$. Furthermore, insulin-treatment may lead to weight gain, fluid retention, higher blood pressure, and increase the risk of hypoglycemia without reducing cardiovascular complications ${ }^{30-32}$. Patients with T2D who require insulin, usually have a poor glycemic and metabolic control, and have a high risk of cardiovascular complications, including HF hospitalisations and death from cardiovascular causes ${ }^{4,33}$. For these reasons, there is an urgent need to reduce the risk profile of insulin-treated patients, while also improving their metabolic components, reduce weight, and decrease the burden of hospitalisations and adverse cardiovascular outcomes. Inhibitors of the sodium glucose co-transporter have many beneficial effects beyond glucose control, and, indeed, may help to reduce weight, control blood pressure, limit sodium retention, reduce hospitalisations (HF-related in particular) and cardiovascular death, counteracting the negative effects of insulin. As shown in the present study, SGLTi reduce cardiovascular complications regardless of insulin treatment. However, the treatment effect was more homogeneous among insulintreated patients leading to consistent reductions of cardiovascular complications, comprising HF hospitalisations and cardiovascular death across multiple populations with T2D, including patients with chronic kidney disease and worsening heart failure ${ }^{7-9,22-24}$. This effect was seen regardless of the type of SGLTi i.e., the SGLT1/2 inhibitor sotagliflozin and the remainder SGLT2 inhibitors had similar effects to reduce cardiovascular events regardless of insulin treatment, but with a more homogeneous effect among insulin-treated patients.

A recently published meta-analysis showed that in placebo-controlled RCTs SGLT2i reduced cardiovascular events and HF hospitalisations, independently of the presence of

217 atherosclerotic cardiovascular disease, kidney function, and glycated hemoglobin levels ${ }^{25}$. It is likely that insulin-treated patients also had higher burden of cardiovascular disease, worse 
kidney and higher glycated hemoglobin levels; however, beyond the higher cardiovascular risk (with potentially larger treatment benefit), insulin-treated patients may experience a more consistent benefit from SGLTi due to the effects and mechanisms associated with insulin administration, such as weight gain and sodium retention that may be counterbalanced by SGLTi. Supporting this hypothesis, smaller "mechanistic" studies testing the effects of SGLT2i among insulin-treated patients also showed a beneficial effect of these agents. For example, a subsample of patients enrolled in the CANVAS-Program and receiving insulin at baseline were randomized to receive placebo ( $n=690)$, canagliflozin $100 \mathrm{mg}$ ( $n$ =692), or canagliflozin $300 \mathrm{mg}(\mathrm{n}=690)$. Compared with the placebo both canagliflozin doses reduced glycated hemoglobin, fasting plasma glucose, body weight and blood pressure ${ }^{15}$. inadequately controlled T2D on basal insulin ${ }^{16,18}$ and obese difficult-to-treat patients with T2D requiring high doses of insulin ${ }^{17}$. It should be noted, that SGLT2i also reduced insulin requirements without increased risk of hypoglycemia ${ }^{17-19}$.

\section{Limitations}

Several limitations are acknowledged. Differences in eligibility criteria, patient characteristics, trial duration, and outcome definition may have affected the treatment effects and increased heterogeneity in the meta-analysis. However, evidence of some heterogeneity was only found among patients without background insulin treatment. The trials here reported, randomised patients to either a SGLTi or placebo but insulin treatment was not part of the randomisation procedure; therefore, these subgroup analyses should be regarded with caution because the treatment effect may be influenced by insulin itself, but also by the patients ' characteristics, their risk profile, comorbidities (including heart failure and chronic kidney disease) or the quality of healthcare. Notwithstanding, the homogeneity 
243 of effect across trials among insulin-treated patients suggests that SGLTi are a beneficial

244 therapy for these patients. For purposes of the present analysis, we were limited to

245 outcomes that had been reported by subgroups of insulin use and some outcome

246 definitions varied between trials (e.g., time-to-first and total heart failure hospitalisations),

247 however the treatment effects were similar across outcomes and this is not a major source

248 of bias in our report. The number needed-to-treat to benefit is not reported because the

249 data required for a reliable calculation of this estimate was not available in all trials.

250

251 Conclusions

252 The present meta-analysis shows that SGLTi reduce cardiovascular events regardless of

253 insulin use. However, the treatment effect is more homogeneous among insulin-treated

254 patients, supporting the use of SGLTi for the treatment of patients with T2D requiring insulin

255 for glycemic control.

256

257 Disclosures

258 J.P.F. is consultant for Boehringer Ingelheim. All the other authors have no relevant conflicts of interest to report.

260

261 Acknowledgments

262 A.C.Oliveira is supported by FCT-Fundação para a Ciência e a Tecnologia, FSE-Fundo Social

263 Europeu, Portugal 2020, NORTE 2020-Programa Operacional Regional do Norte, Centro

264 2020-Programa Operacional Regional do Centro, Alentejo 2020-Programa Operacional

Regional do Alentejo (UI/BD/150647/2020). 
267 Fundo Social Europeu, NORTE 2020-Programa Operacional Regional do Norte, NORTE-08268 5369-FSE-000024-Programas Doutorais.

269

270

271 
1. Herman, M. E.; O'Keefe, J. H.; Bell, D. S. H.; Schwartz, S. S., Insulin Therapy Increases Cardiovascular Risk in Type 2 Diabetes. Prog Cardiovasc Dis 2017, 60 (3), 422-434.

2. MacDonald, M. R.; Petrie, M. C.; Varyani, F.; Ostergren, J.; Michelson, E. L.; Young, J. B.; Solomon, S. D.; Granger, C. B.; Swedberg, K.; Yusuf, S.; et al. Impact of diabetes on outcomes in patients with low and preserved ejection fraction heart failure: an analysis of the Candesartan in Heart failure: Assessment of Reduction in Mortality and morbidity (CHARM) programme. Eur Heart $J$ 2008, 29 (11), 1377-85.

3. Laing, S. P.; Swerdlow, A. J.; Slater, S. D.; Burden, A. C.; Morris, A.; Waugh, N. R.; Gatling, W.; Bingley, P. J.; Patterson, C. C., Mortality from heart disease in a cohort of 23,000 patients with insulin-treated diabetes. Diabetologia 2003, 46 (6), 760-5.

4. Rossello, X.; Ferreira, J. P.; McMurray, J. J.; Aguilar, D.; Pfeffer, M. A.; Pitt, B.; Dickstein, K.; Girerd, N.; Rossignol, P.; Zannad, F. Impact of insulin-treated diabetes on cardiovascular outcomes following high-risk myocardial infarction. Eur Heart J Acute Cardiovasc Care 2019, 8 (3), 231-241.

5. Braffett, B. H.; Dagogo-Jack, S.; Bebu, I.; Sivitz, W. I.; Larkin, M.; Kolterman, O.; Lachin, J. M., Association of Insulin Dose, Cardiometabolic Risk Factors, and Cardiovascular Disease in Type 1 Diabetes During 30 Years of Follow-up in the DCCT/EDIC Study. Diabetes Care 2019, 42 (4), 657-664. 6. Holden, S. E.; Jenkins-Jones, S.; Morgan, C. L.; Schernthaner, G.; Currie, C. J., Glucoselowering with exogenous insulin monotherapy in type 2 diabetes: dose association with all-cause mortality, cardiovascular events and cancer. Diabetes Obes Metab 2015, 17 (4), 350-62.

7. Zinman, B.; Wanner, C.; Lachin, J. M.; Fitchett, D.; Bluhmki, E.; Hantel, S.; Mattheus, M.; Devins, T.; Johansen, O. E.; Woerle, H. J.; et al. Empagliflozin, Cardiovascular Outcomes, and Mortality in Type 2 Diabetes. N Engl J Med 2015, 373 (22), 2117-28.

8. Neal, B.; Perkovic, V.; Mahaffey, K. W.; de Zeeuw, D.; Fulcher, G.; Erondu, N.; Shaw, W.; Law, G.; Desai, M.; Matthews, D. R., Canagliflozin and Cardiovascular and Renal Events in Type 2 Diabetes. N Engl J Med 2017, 377 (7), 644-657.

9. Wiviott, S. D.; Raz, I.; Bonaca, M. P.; Mosenzon, O.; Kato, E. T.; Cahn, A.; Silverman, M. G.; Zelniker, T. A.; Kuder, J. F.; Murphy, S. A.; et al. Dapagliflozin and Cardiovascular Outcomes in Type 2 Diabetes. N Engl J Med 2019, 380 (4), 347-357.

10. Perkovic, V.; Jardine, M. J.; Neal, B.; Bompoint, S.; Heerspink, H. J. L.; Charytan, D. M.; Edwards, R.; Agarwal, R.; Bakris, G.; Bull, S.; et al. Canagliflozin and Renal Outcomes in Type 2 Diabetes and Nephropathy. N Engl J Med 2019, 380 (24), 2295-2306.

11. Zannad, F.; Ferreira, J. P.; Pocock, S. J.; Anker, S. D.; Butler, J.; Filippatos, G.; Brueckmann, M.; Ofstad, A. P.; Packer, M., SGLT2 inhibitors in patients with heart failure with reduced ejection fraction: a meta-analysis of the EMPEROR-Reduced and DAPA-HF trials. Lancet 2020.

12. Zelniker, T. A.; Wiviott, S. D.; Raz, I.; Im, K.; Goodrich, E. L.; Bonaca, M. P.; Mosenzon, O.; Kato, E. T.; Cahn, A.; Furtado, R. H. M.; et al. SGLT2 inhibitors for primary and secondary prevention of cardiovascular and renal outcomes in type 2 diabetes: a systematic review and meta-analysis of cardiovascular outcome trials. Lancet 2019, 393 (10166), 31-39.

13. Neuen, B. L.; Young, T.; Heerspink, H. J. L.; Neal, B.; Perkovic, V.; Billot, L.; Mahaffey, K. W.; Charytan, D. M.; Wheeler, D. C.; Arnott, C. et al. SGLT2 inhibitors for the prevention of kidney failure in patients with type 2 diabetes: a systematic review and meta-analysis. Lancet Diabetes Endocrinol 2019, 7 (11), 845-854.

14. Ferreira, J. P.; Fitchett, D.; Ofstad, A. P.; Kraus, B. J.; Wanner, C.; Zwiener, I.; Zinman, B.; Lauer, S.; Rossignol, P.; Zannad, F., Empagliflozin for Patients with Presumed Resistant Hypertension: a Post Hoc Analysis of the EMPA-REG OUTCOME Trial. Am J Hypertens 2020. 15. Neal, B.; Perkovic, V.; de Zeeuw, D.; Mahaffey, K. W.; Fulcher, G.; Ways, K.; Desai, M.; Shaw, W.; Capuano, G.; Alba, M.; et al. Efficacy and safety of canagliflozin, an inhibitor of sodium- 
glucose cotransporter 2, when used in conjunction with insulin therapy in patients with type 2 diabetes. Diabetes Care 2015, 38 (3), 403-11.

16. Rosenstock, J.; Jelaska, A.; Zeller, C.; Kim, G.; Broedl, U. C.; Woerle, H. J., Impact of empagliflozin added on to basal insulin in type 2 diabetes inadequately controlled on basal insulin: a 78-week randomized, double-blind, placebo-controlled trial. Diabetes Obes Metab 2015, 17 (10), 936-48. Improved glucose control with weight loss, lower insulin doses, and no increased hypoglycemia with empagliflozin added to titrated multiple daily injections of insulin in obese inadequately controlled type 2 diabetes. Diabetes Care 2014, 37 (7), 1815-23.

18. Wilding, J. P.; Woo, V.; Rohwedder, K.; Sugg, J.; Parikh, S., Dapagliflozin in patients with type 2 diabetes receiving high doses of insulin: efficacy and safety over 2 years. Diabetes Obes Metab 2014, 16 (2), 124-36.

19. Wilding, J. P.; Woo, V.; Soler, N. G.; Pahor, A.; Sugg, J.; Rohwedder, K.; Parikh, S., Longterm efficacy of dapagliflozin in patients with type 2 diabetes mellitus receiving high doses of insulin: a randomized trial. Ann Intern Med 2012, 156 (6), 405-15.

20. Wilding, J. P.; Norwood, P.; T'Joen, C.; Bastien, A.; List, J. F.; Fiedorek, F. T., A study of dapagliflozin in patients with type 2 diabetes receiving high doses of insulin plus insulin sensitizers: applicability of a novel insulin-independent treatment. Diabetes Care 2009, 32 (9), 1656-62. 21. Liberati, A.; Altman, D. G.; Tetzlaff, J.; Mulrow, C.; Gøtzsche, P. C.; loannidis, J. P.; Clarke, M.; Devereaux, P. J.; Kleijnen, J.; Moher, D., The PRISMA statement for reporting systematic reviews and meta-analyses of studies that evaluate healthcare interventions: explanation and elaboration. Bmj 2009, 339, b2700.

22. Bhatt, D. L.; Szarek, M.; Steg, P. G.; Cannon, C. P.; Leiter, L. A.; McGuire, D. K.; Lewis, J. B.; Riddle, M. C.; Voors, A. A.; Metra, M. et al. Sotagliflozin in Patients with Diabetes and Recent Worsening Heart Failure. N Engl J Med 2020.

23. Bhatt, D. L.; Szarek, M.; Pitt, B.; Cannon, C. P.; Leiter, L. A.; McGuire, D. K.; Lewis, J. B.; Riddle, M. C.; Inzucchi, S. E.; Kosiborod, M. N. et al. Sotagliflozin in Patients with Diabetes and Chronic Kidney Disease. N Engl J Med 2020.

24. Cannon, C. P.; Pratley, R.; Dagogo-Jack, S.; Mancuso, J.; Huyck, S.; Masiukiewicz, U.; Charbonnel, B.; Frederich, R.; Gallo, S.; Cosentino, F.; et al. Cardiovascular Outcomes with Ertugliflozin in Type 2 Diabetes. N Engl J Med 2020, 383 (15), 1425-1435.

25. McGuire, D. K.; Shih, W. J.; Cosentino, F.; Charbonnel, B.; Cherney, D. Z. I.; Dagogo-Jack, S.; Pratley, R.; Greenberg, M.; Wang, S.; Huyck, S.; et al. Association of SGLT2 Inhibitors With Cardiovascular and Kidney Outcomes in Patients With Type 2 Diabetes: A Meta-analysis. JAMA Cardiol 2020.

26. Higgins, J. P.; Thompson, S. G., Quantifying heterogeneity in a meta-analysis. Stat Med 2002, 21 (11), 1539-58.

27. Sterne, J. A. C.; Savović, J.; Page, M. J.; Elbers, R. G.; Blencowe, N. S.; Boutron, I.; Cates, C. J.; Cheng, H. Y.; Corbett, M. S.; Eldridge, S. M.; et al. RoB 2: a revised tool for assessing risk of bias in randomised trials. Bmj 2019, 366, 14898.

28. Turnbull, F. M.; Abraira, C.; Anderson, R. J.; Byington, R. P.; Chalmers, J. P.; Duckworth, W. C.; Evans, G. W.; Gerstein, H. C.; Holman, R. R.; Moritz, T. E.; et al. Intensive glucose control and macrovascular outcomes in type 2 diabetes. Diabetologia 2009, 52 (11), 2288-98.

29. Gerstein, H. C.; Miller, M. E.; Byington, R. P.; Goff, D. C., Jr.; Bigger, J. T.; Buse, J. B.; Cushman, W. C.; Genuth, S.; Ismail-Beigi, F.; Grimm, R. H., Jr.; et al. Effects of intensive glucose lowering in type 2 diabetes. N Engl J Med 2008, 358 (24), 2545-59.

30. Intensive blood-glucose control with sulphonylureas or insulin compared with conventional treatment and risk of complications in patients with type 2 diabetes (UKPDS 33). UK Prospective Diabetes Study (UKPDS) Group. Lancet 1998, 352 (9131), 837-53. 
371 31. Knatterud, G. L.; Klimt, C. R.; Levin, M. E.; Jacobson, M. E.; Goldner, M. G., Effects of 372 hypoglycemic agents on vascular complications in patients with adult-onset diabetes. VII. Mortality 373 and selected nonfatal events with insulin treatment. Jama 1978, 240 (1), 37-42.

$37432 . \quad$ Lebovitz, H. E., Insulin: potential negative consequences of early routine use in patients with 375 type 2 diabetes. Diabetes Care 2011, 34 Suppl 2 (Suppl 2), S225-30.

376 33. Shen, L.; Rørth, R.; Cosmi, D.; Kristensen, S. L.; Petrie, M. C.; Cosmi, F.; Latini, R.; Køber, 377 L.; Anand, I. S.; Carson, P. E.; et al. Insulin treatment and clinical outcomes in patients with diabetes 378 and heart failure with preserved ejection fraction. Eur J Heart Fail 2019, 21 (8), 974-984. 
Figure 1. Treatment effects by insulin treatment subgroups

(A) Myocardial infarction, stroke or cardiovascular death

383

384

(B) Heart failure hospitalisation or cardiovascular death

385

386 Legend: In (B) EMPA-REG OUTCOME estimates correspond to cardiovascular death only, 387 DECLARE-TIMI-58 and VERTIS CV estimates correspond to the time-to-first of cardiovascular 388 death or heart failure hospitalisation, and SOLOIST-WHF and SCORED estimates correspond 389 to cardiovascular death or first and recurrent heart failure hospitalisations; REML, restricted 390 maximum likelihood. 